\title{
Renaissance des potagers, naissance d'une profession
}

L'exemple de la ville de Niamey au Niger

The Rebirth of Gardens, the Birth of a Professional Development: The case of Niamey in Niger

\section{Anne Luxereau}

\section{OpenEdition}

\section{Journals}

Édition électronique

URL : http://journals.openedition.org/ethnoecologie/2349

DOI : 10.4000/ethnoecologie.2349

ISSN : 2267-2419

\section{Éditeur}

Laboratoire Eco-anthropologie et Ethnobiologie

\section{Référence électronique}

Anne Luxereau, «Renaissance des potagers, naissance d'une profession », Revue d'ethnoécologie [En ligne], 8 | 2015, mis en ligne le 31 décembre 2015, consulté le 02 mai 2019. URL : http:// journals.openedition.org/ethnoecologie/2349; DOI : 10.4000/ethnoecologie.2349

Ce document a été généré automatiquement le 2 mai 2019.

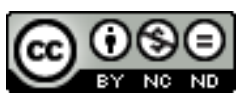

Revue d'ethnoécologie est mis à disposition selon les termes de la licence Creative Commons Attribution - Pas d'Utilisation Commerciale - Pas de Modification 4.0 International. 


\title{
Renaissance des potagers, naissance d'une profession
}

\author{
L'exemple de la ville de Niamey au Niger \\ The Rebirth of Gardens, the Birth of a Professional Development: The case of \\ Niamey in Niger
}

Anne Luxereau

1 Longtemps considérée comme une production tout à fait marginale, l'horticulture en Afrique de l'Ouest et tout particulièrement en ville, concentre depuis les années 1990 l'énergie et les crédits des développeurs publics nationaux et internationaux, des ONG ainsi que l'attention des chercheurs (FAO 2007 ; FAO 2012). Faute de succès manifeste des politiques de développement jusqu'ici mises en œuvre, elle est perçue comme un remède aux crises de subsistance, à la pauvreté, aux inégalités qui se creusent particulièrement dans les villes dont la croissance pose de nouveaux problèmes ${ }^{1}$. Les jardins vivriers urbains sont en vérité peu coûteux et peuvent être fort rentables : à titre d'exemple, dans la région de Dakar, l'agriculture urbaine aurait généré 230 milliards de francs CFA (460 millions d'euros) en 2011 et contribué pour $45 \%$ à l'approvisionnement des urbains (chiffres cités par Koffigan 2012). De nombreuses caractéristiques très positives sont mises largement en avant: ils emploient une main d'œuvre nombreuse, des adultes oisifs et dans une moindre mesure des femmes seules chefs de ménage qui peuvent ainsi se créer des sources de revenus propres et nourrir leur famille; face au changement climatique prévu, ils modifient profondément les paysages en constituent des espaces verts significatifs en période sèche. Mais, ces caractéristiques ont aussi un revers car l'activité peut se révéler dangereuse et non durable à cause de l'emploi ou du suremploi des eaux profondes ou encore d'eaux polluées et de produits phytosanitaires excessifs et non appropriés (Dan-Badjo et al. 2013).

2 Les recherches empiriques ont montré que le développement rapide et massif de ces jardins potagers, en ville comme en campagne, est en réalité plus un fait endogène que le résultat immédiat d'actions organisées de développement et de vulgarisation dont l'influence a été relativement lente à se faire sentir (Bastin \& Fromageot 2007). La 
diversité de ce maraîchage urbain et péri-urbain est grande. Elle sera ici appréhendée à travers l'exemple de Niamey, capitale du Niger, peu différent dans son essence de l'exemple d'autres métropoles ou de villes secondaires d'Afrique de l'Ouest. Mon propos sera de mettre l'accent sur l'inventivité et la vitalité dont ont fait preuve les acteurs locaux, de plus en plus nombreux et diversifiés, à côté et souvent en amont de ces actions développementalistes.

\section{Une installation et une diversification en fonction de I'histoire}

3 Au Niger les surfaces agricoles sont principalement situées sur les ergs sableux ; elles sont dédiées à l'agriculture pluviale d'abord d'autoconsommation, centrée sur les céréales et les légumineuses. Le jardinage irrigué ou de décrue à vocation essentiellement marchande est pourtant ancien. Il a été localisé jusque dans les dernières décades du $\mathrm{xx}^{\mathrm{e}}$ siècle dans un faible nombre de vallées qui ont été longtemps très peu exploitées et dont les sols argilo-sableux bien alimentés en eau par la nappe phréatique peu profonde, lui étaient propices. Ses productions venaient compléter celles de l'agriculture pluviale: plantes condimentaires, tomates et courges pour les sauces, spécialités jouissant d'une renommée régionale comme le tabac de Madaroumfa, le henné de Maradi, ou encore du coton, du riz, du blé irrigué, quelquefois de l'orge. Les premiers explorateurs européens ont mentionné ces jardins à la fin du xix ${ }^{e}$ siècle, déjà à proximité de centres urbains ou de circuits commerciaux régionaux. Au début du $\mathrm{xx}^{\mathrm{e}}$ siècle, les potagers des missionnaires, les jardins destinés à l'alimentation des militaires et des fonctionnaires coloniaux, les jardins d'essai de la recherche agronomique, les jardins-parcs des grands commerçants occidentaux, ont contribué à une diversification des implantations, mais surtout en faveur de l'urbain ou du périurbain. Ils ont aussi déjà un peu transformé les productions ${ }^{2}$ : on leur doit par exemple la création, par des ouvriers de la station agronomique de Tarna, d'un périmètre maraîcher longtemps spécialisé dans la production de légumes à destination des Européens de la ville voisine de Maradi, ou encore l'introduction de la pomme de terre dite aujourd'hui d'Agadez.

4 Jusque vers la fin $\mathrm{du} \mathrm{xx}^{\mathrm{e}}$ siècle, les jardins maraîchers nigériens (ou ouest-africains) ont été consacrés au vivrier marchand (Chaléard 1996) ou à des cultures déjà presque entièrement commercialisées (oignons, piments, poivron, courge, manioc, tomates, ...) : "même si le mil suffit à nous nourrir, les jardins, c'est de l'argent. On mange le manioc et on le vend ${ }^{3}$ ». La saison maraîchère courait - et court encore dans nombre de cas - après celle de l'agriculture pluviale, pendant la saison sèche d'octobre à mai, et leur production était considérée comme secondaire aux céréales. Ainsi un grand nombre de jardins n'étaient mis en culture que lorsqu'il était besoin d'avoir des revenus complémentaires, lors de mauvaises récoltes ou encore lorsque des hommes voulaient amasser un pécule pour se marier ou pour partir en pèlerinage. Pour exemple, dans le département de Tahoua, B. Perriaux (1975) signale qu'entre 1969 et 1973, début des années sèches, le nombre de jardins a augmenté de $41 \%$, dont une moitié au pic de la sécheresse de 1972-73. De même sur le site de Falki, proche de la ville de Mirria, M. Waziri Mato (2000) note que la moitié des jardiniers exercent cette activité depuis leur enfance et qu'en 1973-74 puis en 1984-85 - c'est-à-dire des années de grandes pénuries alimentaires - le site s'est agrandi avec de fortes arrivées de villageois voisins et de sinistrés déplacés. Malgré une évolution en dents de scie, les jardins maraîchers ont progressé un peu 
partout mais leur période d'explosion se situe plus spécifiquement dans le dernier quart $\mathrm{du} \mathrm{Xx}^{\mathrm{e}}$ siècle, au Niger comme dans pratiquement toute l'Afrique de l'Ouest. Au Niger, leur progression s'est en effet accélérée à partir de 1984 dans la foulée d'une décennie de déficit pluviométrique qui a culminé cette année-là, de la saturation foncière, du développement urbain considérable, ainsi que du désengagement des bailleurs internationaux dans le soutien à l'agriculture (Waziri Mato op. cit. ; Fromageot 2008). Les agriculteurs confrontés depuis le début de la décennie 1970 à des déficits répétés puis chroniques de production ${ }^{4}$ ont réutilisé des outils et des savoirs hérités. La possibilité de trouver en ville un marché vivrier attractif, a favorisé l'agrandissement et la pérennisation de zones de jardins ainsi que la migration de ruraux, nigériens ou étrangers, vers ces sites. Cette activité faisant partie d'un fond anti-crise s'est muée en activité permanente.

Les anciennes agglomérations, villages, bourgs ou cités-État, se sont développées sur les champs qui les jouxtaient, par expropriation longtemps sans contrepartie. Autour d'un centre souvent très dense où le végétal a peu de place (mais où un élevage 'de case' peut se développer), l'urbanisation inachevée, qu'elle soit spontanée ou résulte d'un zonage administratif, empiète aujourd'hui encore sur ces champs. L'espace périurbain agricole a ainsi été englobé ou repoussé mais de grandes parcelles non encore bâties sont toujours cultivées, créant une ceinture agricole essentiellement céréalière et d'élevage qui mite l'intérieur même des limites incertaines de la ville. La production maraîchère, l'arboriculture fruitière, la floriculture (en hors sol) sont tributaires d'un accès à l'eau et donc plus groupées. En situation urbaine et périurbaine, on les trouve aujourd'hui le long de cours d'eau permanents ou temporaires, de canaux ou de collecteurs d'eaux usées, sur des délaissés de la ville comme des bords des routes, des pentes, des zones inondables ou sur les terres agricoles des anciens villages devenus des quartiers urbains. Leur statut foncier est variable : les parcelles sont en majorité utilisées par leurs propriétaires mais d'autres le sont par des locataires ou encore par des squatteurs.

6 Trois grands types de jardins peuvent être distingués en fonction de leur localisation, de leur taille et des objectifs des jardiniers. Dans leur grande majorité, les parcelles orientées sur une production commerciale, ne sont pas bien grandes, 0,2 à 0,38 hectare selon Djibo (2013) mais des jardins d'un autre type se déploient sur des parcelles de 2-3 hectares. Ils ne sont pas situés dans les interstices urbains mais, un peu à l'écart de la ville dense, sur des parcelles récemment achetées par des fonctionnaires, des notables (souvent des commerçants nouveaux riches) des personnes qui peuvent n'avoir aucune racine locale ou même nationale (des Libyens par exemple). Leur ampleur, leur agencement souvent avec une construction de réception (une sorte de gloriette) et leur utilisation décrite en termes de loisir, témoignent que si la rentabilité économique est bien recherchée (production potagère et fruitière de rapport en saison sèche, production céréalière en saison des pluies...), ils constituent aussi le signe nouveau de l'appartenance à une catégorie sociale nantie. Il faut enfin mentionner les quelques planches de légumes et les arbres fruitiers disséminés dans les cours des maisons des quartiers résidentiels qui sont loin d'assurer une production autre que minime mais qui, tout en rappelant les anciens 'jardins de case' des femmes rurales, soulignent l'intérêt nouveau des urbains pour cette activité ${ }^{5}$.

7 La dilatation et la densification de la ville mordent sur ces espaces et constituent une véritable menace mais n'ont nullement fait disparaître les différentes formes d'agriculture et d'élevage qui se recomposent diversement : selon L. Andres et P. Lebailly 
(2011), 43 \% de la population de Niamey avait une activité agricole en 2008. Parallèlement à cette croissance urbaine, « les progrès considérables des moyens de communication [...], en réduisant les distances, parfois en les abolissant, ont radicalement changé les rapports à l'espace" (Pélissier 2000). Cela est particulièrement net en matière de jardins maraîchers, où l'espace péri-urbain se révèle être à géométrie variable et recomposé par les acteurs au gré des opportunités, souvent de plus en plus loin des villes. Quelques exemples illustrent bien cette délocalisation plus ou moins lointaine des centres de production et de consommation urbaine. Après la sécheresse de 1973, un groupe Peul ayant perdu l'ensemble de son troupeau, s'est sédentarisé à une vingtaine de kilomètres de la ville et s'est reconverti dans la production de feuilles fraîches très prisées de Moringa $^{6}$ qui sont acheminées quotidiennement sur les marchés de la capitale. De son côté Stéphane Bastin (2009) a étudié l'essor d'une production maraîchère féminine (rare au Niger) qui achalande la ville située à une cinquantaine de kilomètres de là. Enfin la région de Zinder, deuxième ville du Niger, située à plus de 900 kilomètres de la capitale produit $64 \%$ des salades du pays (RGAC 2008). Cette "nouvelle forme d'agriculture urbaine loin de la ville», selon l'expression d'Audrey Fromageot (op. cit.), n'est pas spécifique au Niger ; elle analyse ainsi le maraîchage sénoufo de Côte d'Ivoire, spécialisé dans la production de légumes fragiles qui sont expédiés à 400 kilomètres de là, dans la capitale. Bien que le maraîchage urbain et péri-urbain soit très axé sur le frais, ses limites sont ainsi devenues incertaines et la relation ville campagne s'affirme comme étant multi scalaire $^{7}$. Son essor est le résultat d'initiatives de nombreux paysans qui tirent rapidement parti de la conjoncture offerte par la croissance d'une demande urbaine de produits vivriers variés, dans la mesure où les conditions de vente et l'accès aux marchés sont aisés, c'est-à-dire près des villes et le long des grands axes de communication (Pélissier op. cit.). Mamman Waziri Mato (op. cit.) a bien noté qu'au Niger, les sites périurbains sont essentiellement potagers, ceux qui sont enclavés produisent des légumes qui se conservent tandis que ceux qui sont éloignés des villes mais situés sur des axes de circulation sont mixtes.

\section{Des pratiques innovant en permanence}

8 Le temps où l'objectif du jardinage était de produire une récolte annuelle complémentaire de l'agriculture pluviale, est dans bien des endroits à peu près révolu. À Niamey, si l'on met de côté les parcelles situées en bordure du fleuve Niger qui sont inondées par la crue plusieurs mois par an, les autres tendent à être cultivées en permanence. La rotation des espèces y est la règle. Les salades (récoltées avant de pommer et de monter) ainsi que les carottes poussent rapidement et se succèdent de janvier à juin. Le chou, la carotte, la tomate et la salade se vendent bien en fin de saison des pluies ainsi qu'en période de ramadan et sont donc plantés en conséquence. Les espèces de saison fraîche (tomate, carotte, chou, ...) sont remplacées par des courgettes, des melons et pastèques en saison plus chaude puis laissent la place aux légumes-feuilles anciens, à la menthe et aux épices à thé, au gombo, qui supportent mieux les pluies. Des céréales destinées à l'autoconsommation, occupent enfin les surfaces libérées en fin de saison sèche. Les jardiniers choisissent leurs variétés pour étaler la récolte: les carottes 'touchon' peuvent être remplacées par des 'nantaises améliorées' qui poussent en période chaude; le 'chou cabus', plus précoce que le 'chou de Milan' peut constituer un légume primeur mais le 
second est apprécié en permanence par les cuisinières; le 'chou sahel' peut être décalé des deux premiers.

Les semences des légumes de type européen ont longtemps été achetées auprès des grandes maisons européennes (Clause, Vilmorin, ...). Elles sont en concurrence avec celles que chaque jardinier produit sur une petite planche réservée à cet effet (notamment pour les oignons et les diverses variétés de laitues et de scaroles), et surtout avec celles des nouvelles coopératives locales et les entreprises semencières créées dans la sous-région. L'une d'entre elles, la Société TROPICA SEM a été constituée en 1985 à Dakar par d'anciens chercheurs de la recherche agronomique postcoloniale; elle diffuse directement ou par l'intermédiaire de coopératives, d'associations ou de boutiques d'intrants, des semences de variétés légumières adaptées aux conditions pédoclimatiques locales. L'antenne nigérienne de l'International Crops Research Institute for the Semi-Arid Tropics conseille en particulier un jeune jardinier et lui a fourni de nouveaux fruitiers (pommes-goyave, figuier européen) jusqu'à présent inconnus dont il vend les fruits aux hôteliers locaux et dont il diffuse à son tour des greffons.

La palette d'espèces citées jusqu'à présent fait la place belle aux légumes de type européen surreprésentés jusque dans les années 1980 pour répondre à la demande des expatriés alors très nombreux. Les noms de ces légumes, salati, betaviya, romen ou skarol, karot, shu, tomater, témoignent de leur faible profondeur historique mais aussi de leur insertion dans les cuisines locales. Certains ont quasiment disparu (comme les poireaux et en grande partie les haricots verts ou les échalotes) mais beaucoup d'autres ont en effet été incorporés dans la diète quotidienne locale. Dans la représentation de la santé, l'alimentation tient une grande place et le cru, assimilé aux vitamines et à la force, est désormais prisé. Sur les marchés, des femmes proposent ainsi des salades composées de laitues, d'oignons et de tomates assaisonnées avec du tourteau d'arachide et du piment, et de jeunes marchands ambulants sillonnent les rues avec des grandes cuvettes remplies de carottes à croquer. D'autres ont pris une place majeure dans les recettes anciennes à côté ou en remplacement de brèdes locales de cueillette : le chou entre ainsi dans la confection des bonnes sauces et il est également vendu bouilli en en-cas sur les marchés à côté des feuilles de Balanites aegyptiaca, d'Hibiscus sabdarifa et de diverses corètes. Un retour au goût du terroir à valeur identitaire, aux plantes locales peu onéreuses, est aussi manifeste et bien signalé dans d'autres contextes nationaux (Pélissier op. cit.). Au Niger, les aubergines violettes (yalon nasara, l'aubergine des Blancs) sont de plus en plus concurrencées par les aubergines africaines (yalo) qui se mangent cuites ou crues; les plantes à sauce et les légumes-feuilles (quatre variétés de corètes, cinq d'amarantes, des Cleome gynandra, de l'oseille de Guinée...) deviennent des productions jardinières de plus en plus courantes ainsi que la menthe et les condiments pour le thé (Anethum graveolens); les migrants burkinabés et togolais amateurs de sauces au goût un peu amer ont popularisé le Vernonia amygdalina ainsi que plusieurs espèces de Solanum. Un certain nombre de jardiniers (surtout des Burkinabés) cultivent essentiellement ces légumesfeuilles qui demandent peu d'entretien et rapportent bien. Certains d'entre eux introduisent des variétés importées de chez eux, aux larges feuilles, à côté des variétés locales dont des spontanées naguère plantes de cueillette. Parmi ces annuelles, les espèces et les variétés semées et voulues côtoient ainsi celles qui se ressèment d'un an sur l'autre (quelquefois à partir d'un semencier épargné à cet effet) et d'autres qui sont considérées comme sauvages mais comestibles, admises et laissées en accès libre aux femmes du voisinage. 
11 Les façons culturales témoignent de l'inventivité technique des jardiniers, de la transmission rapide des nouveaux savoir-faire mais aussi des liens internationaux qui lient les acteurs. Le caractère intensif des pratiques se marque aussi par la diversification des espèces pérennes et annuelles, dans le jardin et dans les planches. Des fruitiers locaux et surtout exotiques (surtout des papayers qui font peu d'ombre), des baobabs taillés pour être maintenus arbustes, des Moringa, des plants de tabac, de gombo et de sorgho, sont plantés en lisière des planches de légumes et bénéficient des arrosages. La fumure est la règle partout où la crue n'apporte pas de limons ou encore dans les endroits où les eaux usées utilisées ne compensent pas la baisse de fertilité des sols. Un circuit de vente de fumier animal s'est mis en place entre éleveurs locaux et maraîchers et le recours aux engrais minéraux, plus précoce et surtout bien plus massif qu'en agriculture pluviale ${ }^{8}$, est bien établi : aujourd'hui $4 \%$ des surfaces seulement ne sont pas fertilisées (RGAC, op. cit.). Les produits phytosanitaires, qui sont utilisés en abondance et même en surabondance d'après les agronomes, sont en réalité mieux adaptés à la culture industrielle du coton qu'à la production vivrière. Ils sont importés du Nigéria, du Ghana ou du Burkina Faso sous-tendant une filière commerciale transnationale. Depuis peu, certains jardiniers ont appris à utiliser des décoctions de fruits de neem (Azadirachta indica), un arbre introduit pendant la période coloniale en Afrique de l'Ouest. Sur une base composée de cette décoction éventuellement additionnée de savon ou de tabac, chacun bricole (par exemple en ajoutant un peu de pétrole). Pratiquement partout, l'irrigation à partir de canaux ramifiés par ennoiement de petites planches en creux tend à être remplacée par l'arrosage des planches allongées, légèrement en relief. Il est effectué ici avec des arrosoirs (arozoir ou rozoir) confectionnés à Niamey même, par des tôliers-soudeurs qui en ont acquis la technique et qui recyclent de vieilles tôles de voiture ou de toiture. Lorsque les producteurs en ont les moyens, ils utilisent également des motopompes (pompo) destinées à alimenter une réserve permettant de pallier le faible débit de certains puits ou encore l'éloignement du fleuve. De très rares précurseurs utilisent le goutte-à-goutte. 
Figure $1:$ Grand jardin mixte avec fruitiers (papayers, manguiers, dattiers) salades, tomates (plus loin poivrons, carottes, manioc et même fraisiers) ; une gloriette est aménagée sous les grands arbres. Niamey, Banga Bana, février 2008

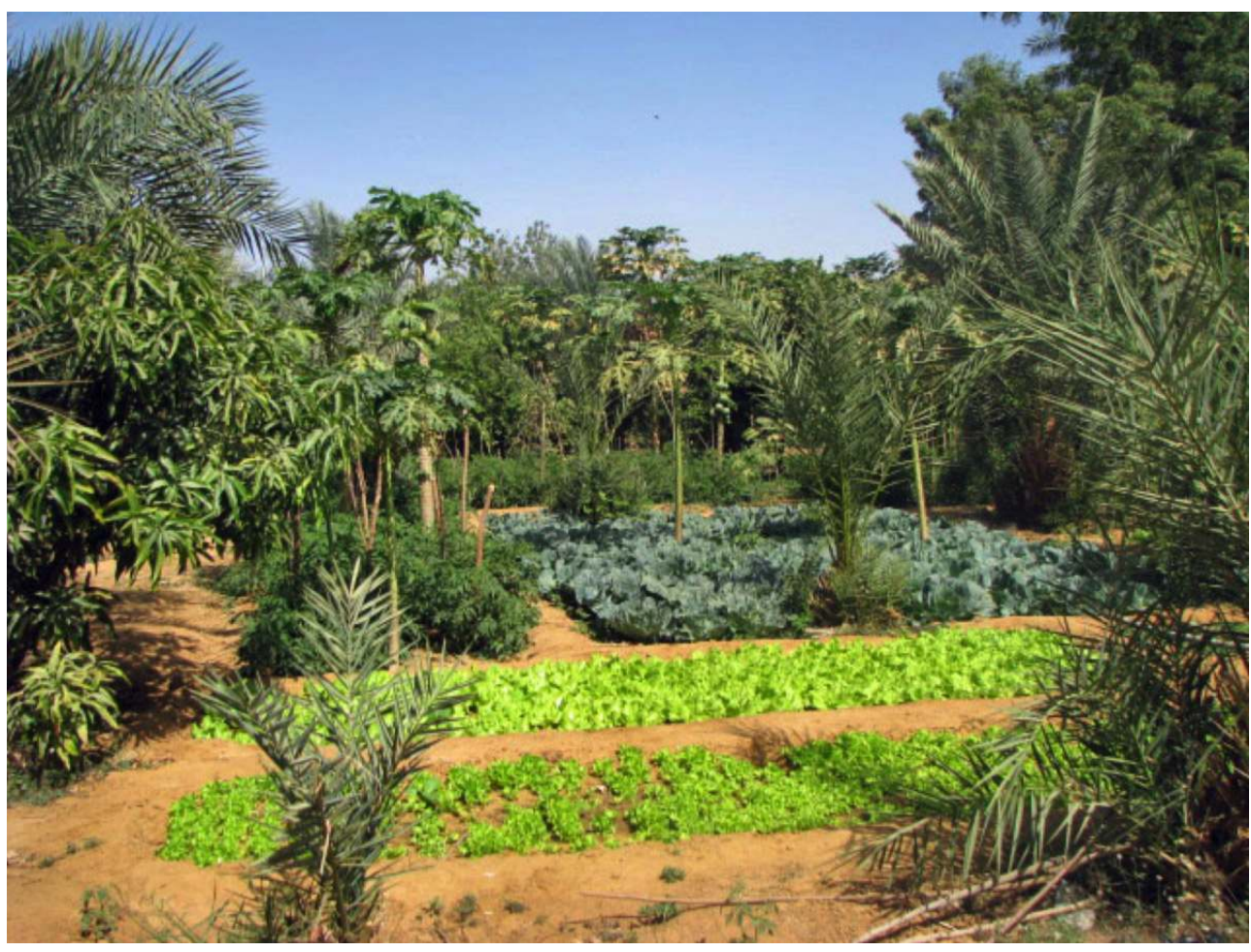

(c) A. Luxereau

Figure 2 : Jardin consacré à la production d'oignons et de Moringa pour la vente. Quelques pieds de manioc, choux, sorgho pour l'autoconsommation. Arrosage par ennoiement des casiers, séparés par des canaux d'amenée de l'eau. Niamey, Lamordé, mars 2007

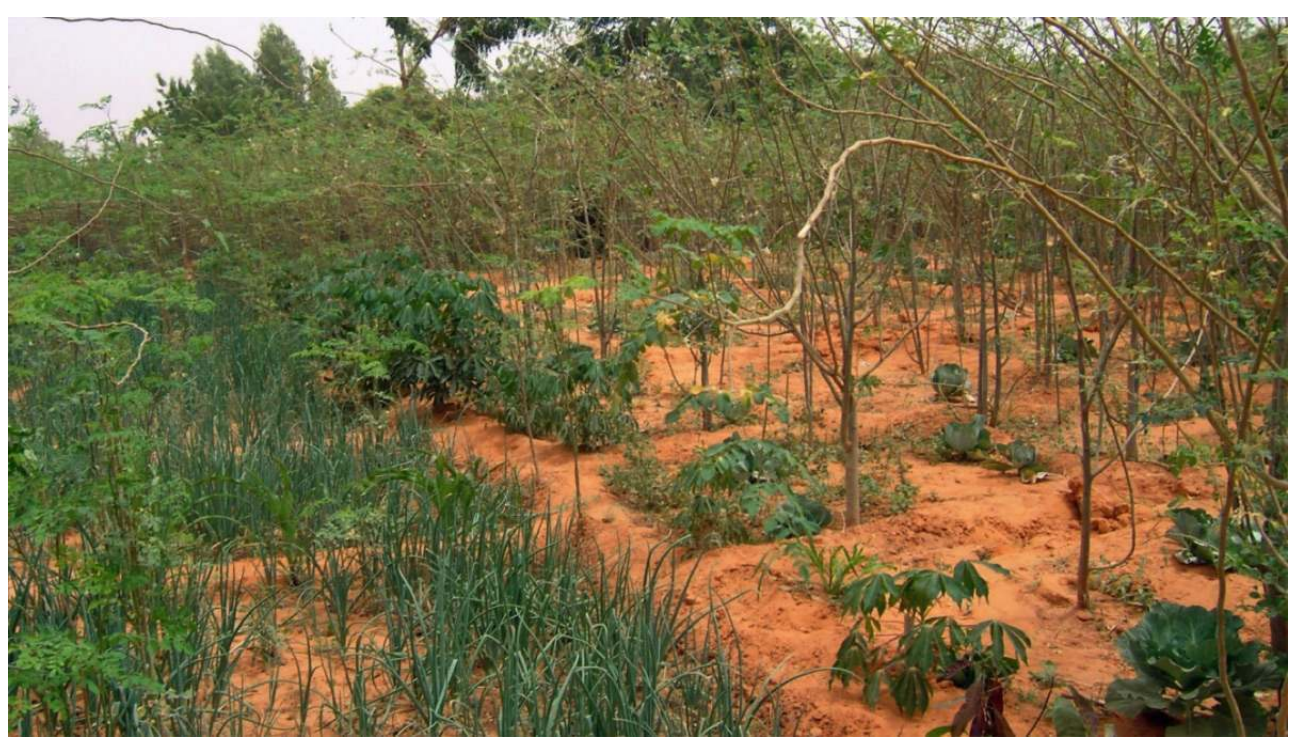

(c) A. Luxereau

12 En somme les jardiniers ont su adapter de façon très dynamique leurs savoir-faire et leurs productions aux opportunités présentées par le développement des marchés urbains et 
les introductions modernes des agronomes. À côté de légumes de type européen, bien installés en ville dans la diète quotidienne, le retour des légumes-feuilles locaux, des plantes condimentaires et aromatiques a augmenté la diversité des produits. Les normes agronomiques ont été appropriées mais sont aussi grandement hybridées et les savoirfaire nouveaux, plus ou moins bricolés, diffusent rapidement. Dans le contexte difficile actuel, l'activité est attractive. Les résultats de ces pratiques sont éloquents avec des rendements moyens de 25 à 30 t/ha pour les tomates, 25 pour les choux, de 15 à plus de 20 pour les carottes et une dizaine pour les salades (Ali Hamidou 2011 ; Djibo op. cit.).

Figure 3 : Jardin centré sur les salades et tomates. À l'ombre des arbres, production de plants de fruitiers en sacs plastiques. Niamey, Gamkallé, corniche du fleuve, décembre 2006

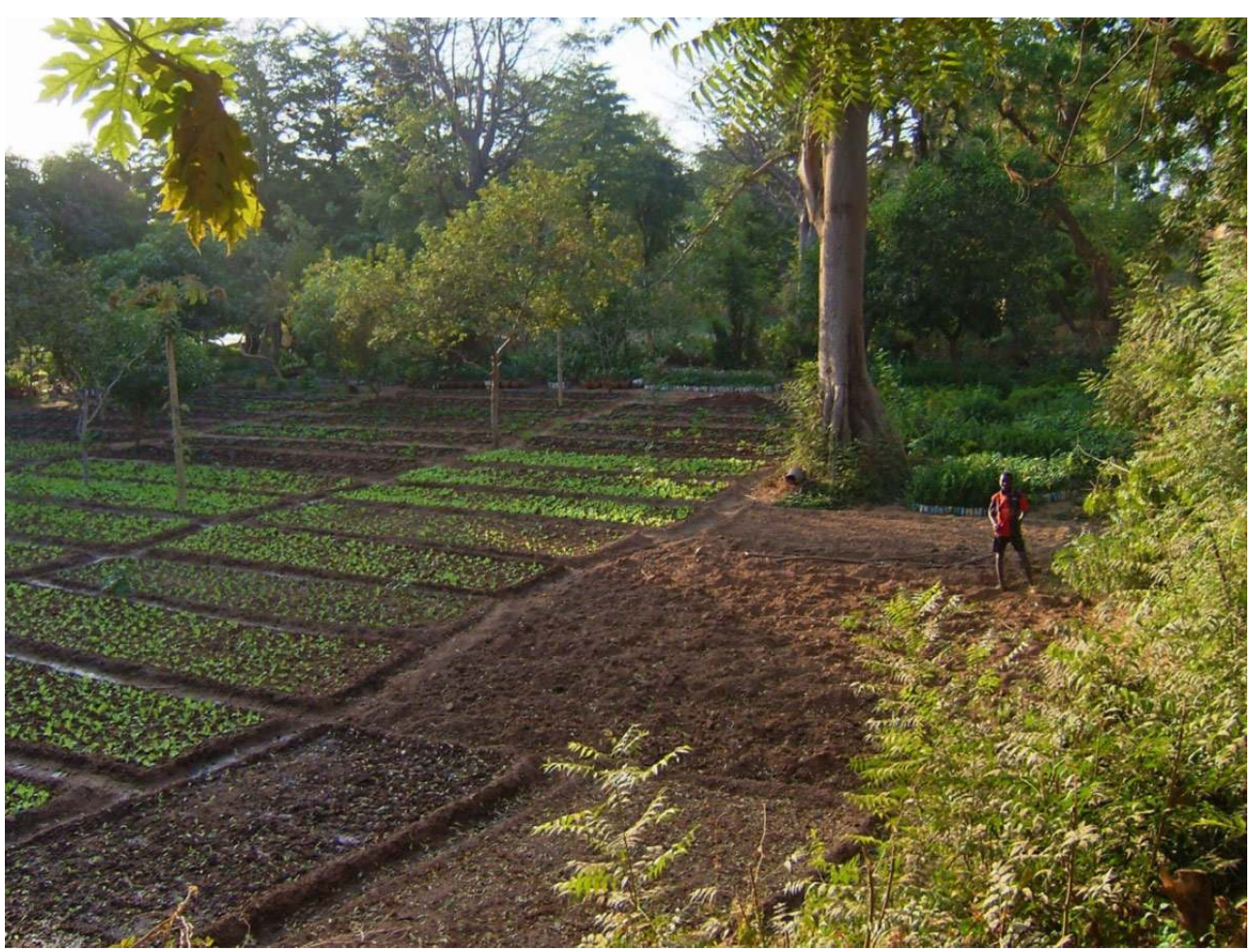

(c) A. Luxereau

\section{La mise en réseau de nouveaux acteurs}

Les femmes sont des agricultrices de saison sèche et pouvaient avoir, dans certaines régions, des lopins consacrés à des cultures de légumes-sauce et plantes thérapeutiques. Le maraîchage est encore le plus souvent sous la maîtrise des hommes. Pourtant, l'exemple des femmes songhay de la vallée du Niger au nord de Niamey, analysé par Stéphane Bastin (op. cit.), prouve que cette spécialisation genrée peut, dans certains contextes, évoluer rapidement. Depuis le milieu des années 1980 et le départ généralisé des maris comme travailleurs migrants pendant la saison sèche, des femmes âgées y ont agrandi et transformé leurs 'jardins de case' en petits champs. Le panel assez divers de plantes cultivées y a évolué en une monoculture de rente, appuyée sur d'anciens réseaux marchands, également féminins, qui maintenant achalandent la ville en oignons, courges et piments. Elles ont rapidement été imitées par des femmes plus jeunes et même récemment par des jeunes filles non mariées qui acquièrent ainsi une certaine 
indépendance économique. À Niamey même, les femmes sont peu présentes et sont plutôt des femmes seules, chefs de famille $.51 \%$ de leur production serait autoconsommée ou échangée alors que celle proportion tombe à $28 \%$ pour les hommes qui orientent plus franchement leur récolte vers le marché (RGAC op. cit.).

En soi, ce travail désormais annualisé a entrainé une professionnalisation des producteurs, surtout de la part de propriétaires exploitants de Niamey qui s'y consacrent à plein temps. Parmi eux, certains ont poussé la spécialisation en cultivant une ou deux espèces, des salades ou carottes par exemple et une très petite production marginale destinée surtout à la consommation familiale (gombo pour les femmes, légumes à sauce). Mais quels que soient les modes de faire valoir, ces jardins maraîchers demandent tous, pour être productifs en permanence, une main d'œuvre importante et constituent ainsi une ressource pour les demandeurs d'emplois. Les propriétaires exploitants ou les locataires de petites parcelles peuvent en effet se charger des façons culturales avec leur famille, par exemple avec les enfants de retour de l'école, à condition d'avoir un accès aisé à une source d'eau et de ne pas se concentrer sur des productions très avides d'arrosage. Dès que la taille des parcelles augmente, le recours à des salariés devient nécessaire, surtout en période de préparation des planches et de pleine récolte, d'octobre à mai. Cette niche de travail a d'abord été exploitée par des migrants burkinabés ou béninois ${ }^{10}$, déjà formés chez eux à ce travail et qui demeurent nombreux. Une seconde vague de migration a été composée d'exodants ruraux nigériens, notamment au moment des pics de sécheresse. Selon H. Djibo (op. cit.) depuis 2010, de jeunes chômeurs originaires de Niamey même, commencent à se faire embaucher. Dans les parcelles de grandes tailles (plusieurs hectares) du bord du fleuve Niger, acquises par des riches commerçants ou des notables locaux, un autre métier est né : celui de 'gardinier' (gardien-jardinier) logé sur place, surveillant le jardin et s'occupant de l'ensemble des travaux, aidé temporairement par d'autres salariés non logés. Alors que les salaires des tâcherons non spécialisés sont médiocres et atteignent rarement le niveau du SMIC nigérien (environ 35 euros par mois), le gardinier bénéficie d'une partie du terrain pour ses propres cultures (ce qui bien souvent lui tient lieu de salaire) et quelquefois gère au quotidien les ventes sur place, ce qui permet souvent au propriétaire de se dire lésé d'une grande partie des revenus, mettant ainsi l'accent sur une finalité ostentatoire plutôt que marchande de ces jardins.

À métier nouveau, nouvelles filières, locales ou transnationales. Celles d'approvisionnement en outillage, semences et plants, en fumier et produits phytosanitaires, qui mettent en relation des personnes aux statuts diversifiés ont déjà été brièvement décrites. La commercialisation des récoltes de ces jardins s'effectue à 90,6 \% à Niamey même (RGAC op. cit.), avec ou sans transformation et mobilise cette fois autant les hommes que les femmes. Les achats sont réalisés directement dans les jardins, au tas ou à la planche levée. Les grossistes sont plutôt masculins et ce sont surtout des hommes qui se chargent du transport jusqu'aux différents marchés ou restaurants de la capitale ${ }^{11}$. Les revendeurs détaillants sont également souvent des hommes. Ils pratiquent ce commerce soit à plein temps en tant que 'tablier', vendant légumes et fruits sur une table-étal dans ces marchés et payant patente, soit à la sauvette en proposant à la sortie des boutiques ou dans les rues une seule espèce de légume ou de fruit dans une brouette ou une grande cuvette protégées par un sac régulièrement humidifié ${ }^{12}$. Les femmes achètent aussi les produits directement dans les jardins (se chargeant souvent même de la récolte), et les revendent au détail ou les transforment souvent elles-mêmes. De nombreuses femmes âgées consacrent ainsi une bonne partie de la journée à vendre des salades, des 
condiments ou des en-cas de légumes sur les marchés de la capitale : en 1987 sur le site du 'petit marché', l'un des plus importants pour la vente de légumes, 228 femmes vendeuses de fruits et légumes étaient présentes à côté de 318 hommes (selon une étude du ministère de l'Agriculture citée par F. Ali Hamidou op. cit.). On trouve parmi les femmes intéressées par ce secteur, des restauratrices, surtout béninoises et togolaises, qui tiennent de petites gargotes de plein air le long des routes importantes, dans les gares routières, à proximité des administrations.

Figure 4 : Une commerçante récolte des feuilles de Moringa. Derrière elle, une planche de salades montées en graines. Niamey, Haro Banda, mars 2005

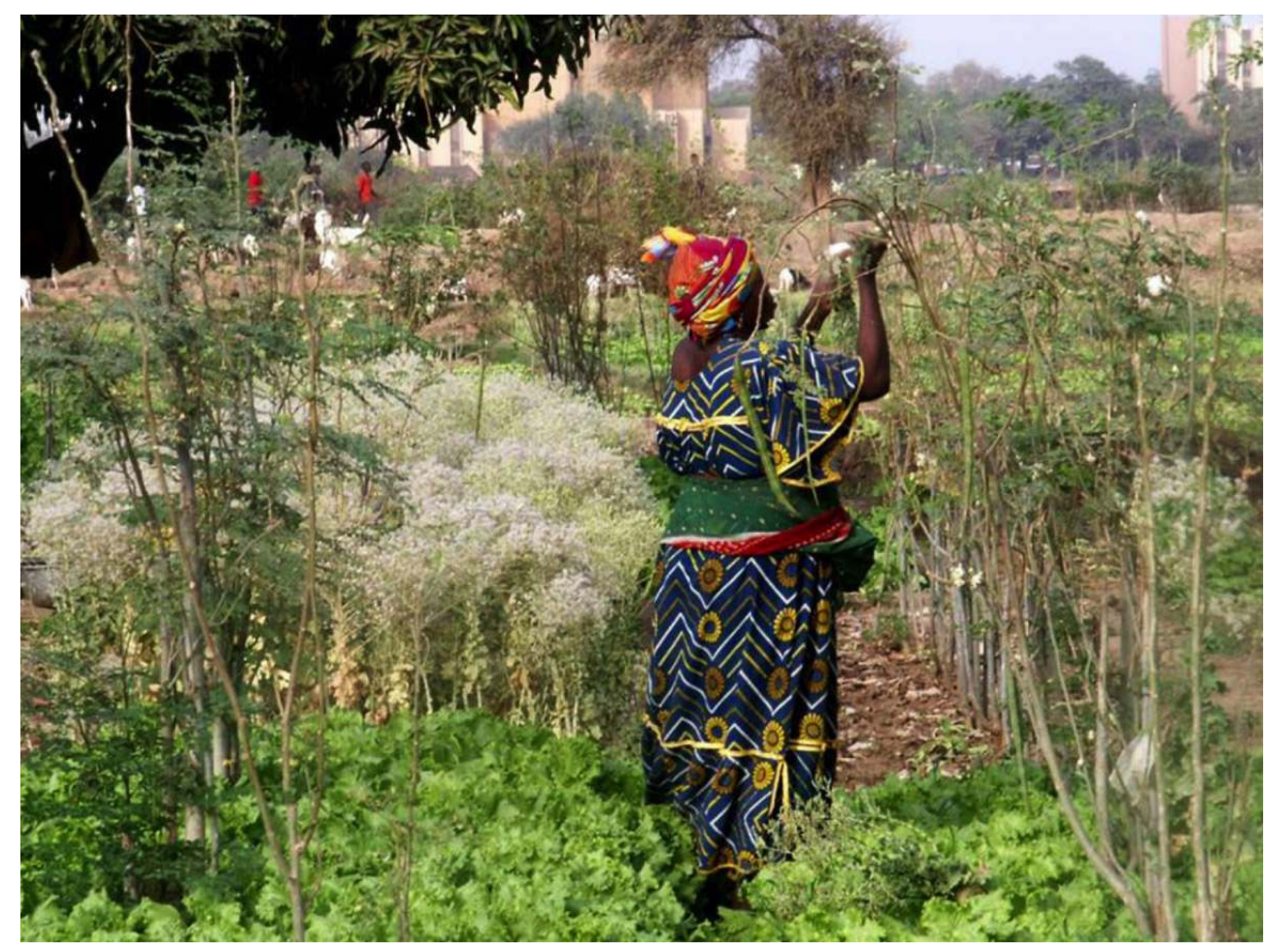

(c) B. Roussel

\section{Une activité attractive à toutes fins}

La rentabilité économique du jardinage urbain et périurbain est une raison majeure de son développement en période normale ou de crise. Mais elle n'est pas atteinte par tous. En 1992, un hectare d'oignons pouvait générer un bénéfice oscillant entre 300000 et 1000000 Francs CFA (soit environ 450 à 1500 euros [Luxereau \& Roussel 1998]). Plus récemment, H. Djibo (op. cit.) note que les producteurs de choux de Niamey sont ceux qui s'en sortent le mieux mais également que si les gros producteurs peuvent avoir des revenus mensuels de 100000 à 130000 Francs CFA (presque le double du salaire actuel des jeunes instituteurs) les plus petits, et parmi eux les locataires, sont tout juste au niveau du seuil de pauvreté (au Niger estimé à 400 Francs CFA/jour, environ 0,6 euros). Les frais de production sont en effet importants en début de saison (location du terrain, main d'œuvre, achat des semences, des plants, des engrais et produits phytosanitaires, du carburant des motopompes, etc.) et il est vital d'éviter le recours à l'emprunt. Ceux qui doivent rembourser un crédit vendent en effet leurs produits au plus vite, généralement 
au plein de la récolte lorsque les cours sont bas or les prix peuvent tripler entre la période de pleine production et celle de pénurie; ceux qui n'ont pu acheter suffisamment d'engrais ou de produits phytosanitaires faute d'argent, ont de maigres récoltes et vendent au rabais des produits pas très beaux.

Cette rentabilité n'est pas non plus un objectif affiché par tous les exploitants et les catégories normatives sont multiples. Il faut tout d'abord se souvenir qu'une part variable des produits est autoconsommée : elle est importante pour les femmes mais du côté des hommes, F. Ali Hamidou (op. cit.) note que, dans son échantillon, l'autoconsommation et le don combinés s'élèvent à $10 \%$ pour les grands producteurs, $13 \%$ pour les producteurs moyens et $19 \%$ pour les petits. Par ailleurs les grandes parcelles achetées récemment sont souvent présentées comme "coûtant plus qu'elles ne rapportent ${ }^{13}$ " bien qu'elles soient menées dans une logique parfaitement spéculative. «Maintenant tous ceux qui ont les moyens veulent un jardin ${ }^{14}$ " pour aller s'y reposer pendant la saison chaude ou les soirs de ramadan en famille ou avec des collègues et des amis, pour pouvoir offrir des fruits encore rares (grenades, figues, fraises, ...) ou des légumes dessaisonnés, pour employer des parents pauvres ou des obligés. La finalité mise en avant est cette fois la représentation et l'entretien des réseaux de sociabilité. Enfin nombre de fonctionnaires moins argentés installent dans la cour de leur maison de minuscules parcelles de salades ou de tomate pour déguster - et faire déguster - ce qu'ils ont eux-mêmes produit et pour la qualité biologique de ces légumes. Ces cas qui ne sont évidemment pas les plus nombreux, traduisent bien l'engouement pour cette activité.

\section{Conclusion : une stratégie ancienne revisitée et réinventée en métier}

18 Au Niger, le maraîchage urbain et péri-urbain est une activité ancienne, inféodée à l'accès à l'eau, qui a souvent été exercée de manière sporadique pour compenser un manque soit d'argent soit de nourriture. La nouveauté vient de sa croissance et de sa pérennité en milieu urbain, péri-urbain ou rural, dans ce pays comme un peu partout en Afrique de l'Ouest. Il témoigne de l'aptitude des agriculteurs à pallier les difficultés chroniques de l'agriculture pluviale avec des stratégies anciennes revisitées mais également à se saisir des opportunités créées par la croissance urbaine et la création d'un réseau de liaisons routières (ou fluviales pour le Niger). Comme auparavant, les agriculteurs diversifient leurs sources de revenus en jouant sur la complémentarité des écosystèmes et des saisons mais une part grandissante d'entre eux se professionnalise. Ces producteurs sont tournés vers la modernité avec des techniques intensives, des espèces et variétés exogènes ou locales qui évoluent et se remplacent les unes les autres en fonction de la demande et des modes. L'engouement pour une alimentation où la part des légumes et des fruits augmente, soutient l'activité de jardiniers urbains ou migrants, nigériens ou étrangers, et qui devrait intégrer de plus en plus de femmes; il permet de pérenniser des flux quelquefois lointains de légumes frais et fragiles et de multiplier les opérateurs de filières souvent transnationales de production et de commercialisation. Cette demande de légumes et de fruits diffuse vers les campagnes qui consomment de plus en plus les nouveaux légumes de type européen mais propagent aussi en retour vers les villes l'ancien goût des légumes et des brèdes locaux. L'essor des jardins maraîchers, en ville et dans l'espace péri-urbain plus ou moins proche de Niamey, ne peut ainsi être dissocié de ce qui se passe désormais dans l'ensemble du monde rural où, malgré des disparités 
régionales, la production maraîchère est partout en forte croissance dès que les conditions agropédologiques et un accès adéquat aux marchés le permettent. Les villes moyennes et les bourgs ont désormais une ceinture potagère et fruitière bien plus pérenne que les ceintures vertes forestières coloniales ou postcoloniales.

L'aptitude des jardiniers à profiter des nouvelles routes et des actions et financements des services techniques de l'État et de la coopération internationale qui multiplient les forages, puits et petits barrages, témoigne bien également qu'on est loin de la représentation archaïsante de systèmes de production vivriers rétifs au changement. Dans le même temps, en investissant les marges, les délaissés, les interstices des espaces urbains et péri-urbains, ces jardiniers font aussi preuve d'un certain talent à contourner les contraintes et investir dans des activités productrices, éventuellement à court terme. Selon la FAO (2012) $40 \%$ des citadins africains produisent des légumes, quelquefois dans des micro-jardins aux modalités totalement novatrices.

\section{BIBLIOGRAPHIE}

Ali Hamidou F. 2011 - Impacts des activités maraîchères sur les revenus des producteurs au Niger : cas du site de Gamakallé. Diplôme de Master complémentaire en économie-sociologie rurales. Université catholique de Louvain, Université de Liège, Gembloux Agro bio tech.

Andres L. \& Lebailly P. 2011 - Peri-urban Agriculture: The Case of Market Gardening in Niamey, Niger. African Review of Economics and Finance 3(1), 69-80 (accessible en version française : Université de Liège, Open Repository and Bibliograpy. http://orbi.ulg.ac.be/ bitstream/2268/126098/1/Article\%200rl\%C3\%A9ans.pdf

Bastin S. 2009 - Diffusion du maraîchage féminin et grands espaces de mobilité dans la vallée du Niger. Techniques \& Culture, Des Choses, des gestes, des mots, 51: 124-143. | 2009, mis en ligne le 11 juin 201. URL : http://tc.revues.org/4668

Bastin S. \& Fromageot A. 2007 - Le maraîchage : révélateur du dynamisme des campagnes sahélosoudaniennes. Belgéo $4:$ 415-427.

Chaleard J.-L. 1996 - Temps des villes, temps de vivres. L'essor du vivrier marchand en Côte d'Ivoire, Paris : Karthala.

Dan-Badjo A.T., Guero Y., Lamso N.D., Barage M., Balla A., Sterckeman T., Evarria G.E. \& Feidt C. 2013 - Évaluation des niveaux de contamination en éléments traces métalliques de laitue et de chou cultivés dans la vallée de Gounti Yena à Niamey, Niger. Journal of Applied Biosciences 67 : http://www.ajol.info/index.php/jab/issue/view/10483

Djibo H. 2013 - L'agriculture urbaine et périurbaine : le maraîchage à Niamey (Niger). Thèse de socioéconomie du développent. Paris, EHESS, $185 \mathrm{p}$.

FAO 2007 - Profitability and sustainability of urban and peri-urban agriculture. Rome, FAO, 108 p. (Agricultural management, marketing and finance occasional Paper ; 19).

FAO 2012 - Pour des villes plus vertes en Afrique. Premier rapport d'étape sur l'horticulture urbaine et périurbaine. Rome, FAO, $116 \mathrm{p}$. 
Fromageot A. 2008 - Le maraîchage marchand dans le nord de la Côte d'Ivoire : une nouvelle forme d'agriculture urbaine loin de la ville? In Vidal R (Dir.) La diversité de l'agriculture urbaine dans le monde, vol. 3 des actes du colloque Les agricultures périurbaines, un enjeu pour la ville. (C) ENSP, Université de Nanterre : 95-108.

INS 2010 - Annuaire statistique des cinquante ans d'indépendance du Niger. République du Niger.

Koffigan E. A. 20/11/2012 - L'horticulture urbaine se développe malgré les difficultés. Inter Press Service News Agency, Sénégal, http://www.ips.org/fr/senegal-lhorticulture-urbaine-se-developpemalgre-les-difficultes/

Luxereau A. \& Roussel B. 1998 - L'exploitation des bas-fonds en pays haoussa (Niger) : permanence et innovation. In Chastanet M. (Dir.) Plantes et paysages d'Afrique. Une histoire à explorer . Paris, Karthala ; CRA : 551-571.

Pélissier P. 2000 - Les interactions rurales - urbaines en Afrique de l'Ouest et Centrale Bulletin de l'APAD [En ligne], 19 | 2000, mis en ligne le 12 juillet 2006. Consulté le 15 juillet 2010. URL : http:// apad.revues.org/ 422

Perrriaux B. 1975 - Les cultures maraîchères dans le département de Tahoua, République du Niger. Mémoire de maîtrise de géographie, Université de Paris X, Nanterre.

Raynaut Cl. 1988 - La culture irriguée en pays haoussa nigérien. Aspects historique, sociaux, techniques. Etudes Rurales 115-116 : 105-128.

RGAC 2008 - Recensement général de l'agriculture et du cheptel 2005/2007, vol IX, horticulture. République du Niger - UE - FAO.

Waziri Mato M. 2000 - Les cultures de contre-saison dans le sud de la région de Zinder (Niger). Thèse de doctorat, Institut de géographie, Université de Lausanne.

\section{NOTES}

1. Entre 1960 (année de l'indépendance) et 2010, la population nigérienne est passée d'un peu plus de 3 millions d'habitants à un peu plus de 15 millions, selon les chiffres officiels. Dans le même temps, la population urbaine a fait un saut de 169000 à 3104000 habitants dont 1222000 résident dans la Communauté urbaine de Niamey (INS 2010).

2. Voir l'article de Dominique Juhé-Beaulaton dans cet ouvrage.

3. Jeune agriculteur de la région de Birnin Konni (1992)

4. Depuis 1960 , le Niger a connu des années d'insécurité alimentaire transitoire liée à la sécheresse et/ou à des invasions acridiennes en 1966/67, 1973/74, 1984/85, 1990/91, 1993/94, 1996/97, 2000/01, 2004/05, 2009/2010. La stagnation des rendements des cultures et la vigoureuse croissance démographique ont contribué à la création d'un déficit alimentaire chronique depuis le début des années 2000. Des programmes de vulgarisation de «cultures de contre-saison " avec création de périmètres horticoles ont été lancés par l'État nigérien entre 1982 et 1987, dont un programme d'urgence après la grande sécheresse de 1984/1985. Des ONG (en particulier les églises missionnaires) ont mené des actions locales ultérieures, souvent de petite envergure.

5. J'exclus les rizières pluviales incluses dans la ville qui s'est étendue sur les deux rives du fleuve Niger, ainsi que les périmètres rizicoles irrigués créés par les coopérations internationales.

6. Le Moringa oleifera est ici utilisé pour ses feuilles mélangées à du couscous (de mil ou d'autres céréales) pour la confection d'un plat très apprécié, le kopto, qui peut être mangé froid sur les marchés. 
7. D'ailleurs, la ville de Niamey a une production de fruits excédentaire (près de 9500 tonnes) qu'elle doit écouler dans sa zone d'influence (Andres \& Lebailly op. cit.).

8. C. Raynaut (1988) signale que les plus gros utilisateurs d'engrais de la région de Maradi ont longtemps été les jardiniers-maraîchers du village proche de Soumarana pourvoyant la ville en légumes frais.

9. Les femmes étaient des agricultrices de saison sèche, et pouvaient avoir, dans certaines régions, des lopins consacrés à des cultures de légumes-sauce et plantes thérapeutiques. Aujourd'hui, selon le Recensement général de l'horticulture (RGAC op. cit.), 18,8 \% de l'ensemble des exploitations horticoles du Niger (toutes catégories confondues) sont gérées par des femmes, avec quelques pointes comme dans le pourtour de la ville de Dosso, où 61,3\% des exploitations horticoles de la région sont aux mains de femmes

10. Les migrants Touaregs des sécheresses ou des guerres possèdent souvent un savoir-faire très fin en la matière. Ils ont été longtemps recrutés en Lybie comme jardiniers mais ne le sont que très peu à Niamey.

11. En zone rurale, de nombreux groupes plus ou moins bien formalisés de producteurs, ont acheté des camionnettes ou des camions pour transporter les produits jusqu'aux marchés d'importance les plus proches.

12. H. Djibo (op. cit.) mentionne aussi les intermédiaires entre les grossistes, des détaillants, certains services publics comme les cantines scolaires et universitaires, l'hôpital, les casernes.

13. Un gros commerçant de Maradi, 1995.

14. Enseignant d'université, 2002.

\section{RÉSUMÉS}

L'horticulture, longtemps marginale, a connu un essor remarquable après les grandes sécheresses culminant dans les années 1985-90, au Niger comme dans les Etats voisins. En ville et dans des régions bien reliées par des circuits de commercialisation, les producteurs de légumes et de fruits se sont saisi des marchés urbains en expansion. Ils ont su hybrider, de façon très dynamique, les techniques anciennes et celles introduites par l'agronomie moderne. Ces producteurs se sont professionnalisés. Ils sont tournés vers la modernité avec des techniques intensives, des espèces et variétés exogènes ou locales qui évoluent et se remplacent les unes les autres en fonction de la demande et des modes. Au Niger comme dans les autres pays d'Afrique de l'Ouest, les villes moyennes et les bourgs ont désormais une ceinture potagère et fruitière modifiant les paysages.

For a long time, Nigerian gardening was a secondary production. It developed after the 1985-90 droughts in Niger and neighboring states of West Africa. In towns and in well-connected areas gardeners settled in growing urban markets. They managed to mix an old know-how to a new one brought by modern agronomy. A new job was born and the producers turned to modernity. They implement intensive practices. They grew local and exogenous species and varieties, the evolution of which is linked to demand and fashion. In Niger as well as in the other West African countries, cities and middle-sized towns have now a green belt of vegetables and fruit gardens that modify landscapes. 
INDEX

Keywords : Africa, Niger, garden, town, know-how Index géographique: Afrique, Niger, Niamey

Mots-clés : jardin, ville, savoir-faire

\section{AUTEUR}

\section{ANNE LUXEREAU}

Ethnologue, MNHN, département Hommes Natures Sociétés, UMR 7206, CP 26, 57 rue Cuvier, 75231 PARIS

luxereau@mnhn.fr 